\title{
TOROIDAL EFFECTS ON PROPAGATION, DAMPING, AND LINEAR MODE CONVERSION OF LOWER HYBRID WAVES
}

BY

D. W. IGNAT

\section{PLASMA PHYSICS LABORATORY}

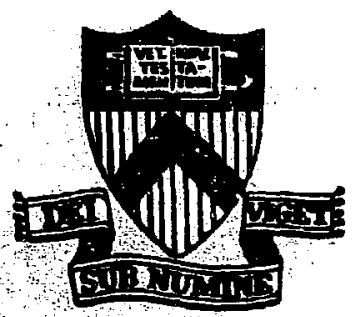

SW,

\section{PRINCETON UNIVERSITY PRINCETON, NEW JERSEY}




\section{NOTICE}

This report was prepared as an account of work sponsored by the United States Government. Neither the United States nor the United States Department of Energy, nor any of their employees, nor any of their contractors, subcontractors, or their employees, makes any warranty, express or implied, or assumes any legal liability or responsibility for the accuracy, completeness or usefulness of any information, apparatus, product or process disclosed, or represents that its use would not infringe privately owned rights.

Printed in the United States of America.

Available from:

National Technical Information Service

U. S. Department of Commerce 5285 Port Royal Road

Springfield, Virginia 22151

Price: Printed Copy \$_*_; Microfiche $\$ 3.00$

*pages

NTIS

$$
1-50
$$

$51-150$

$151-325$

$326=500$

$501-1000$

Selling. Price

$\$ 4.00$

85,45

$\$ 7.60$

$\$ 10.60$

$\$ 13.60$ 
Toroidal Effects on Propagation, Damping, and Linear Mode Conversion of Lower Hybrid Waves

D. W. Ignat

Plasma Physics Laboratory, Princetor, University Princeton, NJ 08544

A common simplifying assumption made in the consideration of radio-frequency heating of tokamaks near the lower hybrid frequency is that the wavelength imposed by the coupling device parallel to the magnetic field is not modified by gradients along the field. In the present calculation, the parallel wavelength is allowed to vary, and important effects are found on wave penetration and damping if the toroidal aspect ratio $\left(\mathrm{R}_{\text {major }} / \mathrm{r}_{\text {minor }}\right)$ is less than $\sim 5$. The calculation shows that heating at the center of a small aspect ratio torus is inhibited by a decrease of $k_{\mid 1}$ if waves are launched at the outside, and that it may be possible to change the plasma current via electron Landau damping with a coupler of symmetric power spectrum by placing the coupler at the top (or bottom) of the torus. 


\section{Theoretical Model}

Understanding of propagation and damping $u$ f the lower hybridwave in nonuniform plasma proceeds from the application of the eikonal (or WKB) method ${ }^{l}$ to some ap sroximation of a kinetic treatment of waves in a magnetized plasma. Field quantities are written

$$
\underline{E}(\underline{r}, t)=\left[\underline{E}_{0}(\underline{r})+\underline{E}_{1}(\underline{r}) \cdots\right] \cdot \exp [i S(\underline{r})-i \omega t]
$$

where, in zero order, spatial variations are contained in $S(\underline{r})$, a rapidly varying quantity lescribing phase fronts of the wave. The result of the method, to zeroth order, is that $\underline{k}=\underline{\nabla} S(\underline{r})$ is the solution of the local dispersion relation $\mathrm{D}(\underline{\mathbf{r}}, \underline{\mathbf{k}}, \omega)=0$ and that the wave evolves in confiquration and $k$ space according to

$$
\begin{aligned}
& \frac{d \underline{r}}{d t}=\frac{-\partial D(\underline{r}, \underline{k}, \omega) / \partial \underline{k}}{\partial D(\underline{r}, \underline{k}, \omega) / \omega}, \\
& \frac{d \underline{k}}{d t}=\frac{+\partial D(\underline{r}, \underline{k}, \omega) / \partial \underline{r}}{\partial D(\underline{r}, \underline{k}, \omega) / \partial \omega} .
\end{aligned}
$$

In higher orders, formulae describing the evolution of the amplitudes $\mathrm{E}_{0}(\underline{r}), \underline{E}_{2}(\underline{r}) \ldots$, are obtained. 1,2 The local dispersion relation can be written

$$
D(\underline{r}, \underline{k}, \omega)=\left|\underline{k} \underline{k}-\underline{\underline{I}} k^{2}+\frac{\omega^{2}}{c^{2}} \underline{\underline{K}}(\underline{\underline{r}}, \underline{k}, \omega)\right|=0
$$

if electromagnetic portions of Maxwell's equations are retained or

$$
D(\underline{r}, \underline{k}, w)=\underline{k} \cdot \underline{\underline{K}}(\underline{r}, \underline{k}, w) \cdot \underline{k}=0
$$


in the electrostatic approximation. Here $\underline{\underline{K}}$ is the hot plasma dielectric temsor as described by Sti..3 Much of the physics of the propagation is found by a "warm-plasma" expansion of $\underline{\underline{K}}(\underline{r}, \underline{k}, \omega)$ in which first-order finite-Larmor-radius effects for ions and electrons are retained. After such an expansion, Eqs. (3) and (4) become, respectively,

$$
\begin{aligned}
D(\underline{\underline{r}}, \underline{k}, \omega)=-\alpha k_{\perp}^{6}+\left[k_{\perp} k_{\perp}^{2}+k_{11}\left(k_{11}^{2}-\frac{\omega^{2}}{c^{2}} k_{\perp}\right)\right] \cdot\left(k_{\perp}^{2}+k_{11}^{2}-\frac{\omega^{2}}{c^{2}} k_{\perp}\right) \\
+\frac{\omega^{2}}{c^{2}} k_{x y}^{2}\left(k_{\perp}^{2}-\frac{\omega^{2}}{c^{2}} k_{11}\right)=0,
\end{aligned}
$$

and

$$
D(\underline{r}, \underline{k}, w)=-\alpha k_{1}^{4}+k_{1}^{2} K_{1}+k_{11}^{2} K_{1}=0
$$

where

$$
\begin{aligned}
& \alpha=3 \frac{\omega_{\mathrm{p} i}^{2}}{\omega^{2}} \frac{v_{\mathrm{T} i}^{2}}{\omega^{2}}+\frac{3}{4} \frac{\omega_{\mathrm{pe}}^{2}}{\omega_{\mathrm{ce}}^{2}} \frac{v_{\mathrm{T} e}^{2}}{\omega_{\mathrm{ce}}^{2}} \\
& K_{\perp}=I+\frac{\omega_{p e}^{2}}{\omega_{c e}^{2}}-\frac{\omega_{\mathrm{p} i}^{2}}{\therefore} ; K_{11}=1-\frac{\omega_{\mathrm{pe}}^{2}}{\omega^{2}} \\
& k_{x y}=\frac{\omega_{p e}^{2}}{\omega \omega_{c e}} \\
& v_{T_{1, e}}^{2}=\frac{k T_{i, e}}{m_{i, e}}
\end{aligned}
$$

and $\omega_{p i}, \omega_{p e}$ ' $\omega_{c e}$ are the two plasma frequencies and the electron cyclotron frequency, respectively. We have assumed inequalities appropriate to the lower hybrid problem $\omega_{c e}^{2} \gg \omega^{2} \gg \omega_{c i}^{2}$, used $\omega_{\mathrm{pe}}^{2} \gg \omega_{\mathrm{pi}}^{2}$, and written $\mathrm{k}_{\mathrm{l}}=\underline{\mathrm{k}} \cdot \underline{\mathrm{B}} /|\underline{\mathrm{B}}|, \mathrm{k}_{\perp}^{2}=|\underline{\mathrm{k}}|^{2}-\mathrm{k}_{\mathrm{l}}^{2}$. 
Equations equivalent to Eq. (5) and Eq. (6) have been used by Stix ${ }^{4}$ and others, 5,6 and are accurate if $\left(\mathrm{k}_{\perp} \mathrm{v}_{\mathrm{T}_{1}} / \omega\right)^{2} \ll 1$ and $\mathrm{k}_{1} \neq 0$.

The wave of primary interest in lower hybrid heating is electrostatic and well described by Eq. (6). However, if $\eta_{11}=k_{1 /} / \omega$ is small enough (less than 2-3), electromagnetic effects become impurtant and $\mathrm{Eq}$. (5) should be used.

The ray equations (1) - (2) and (5) or (6) may be integrated in Cartesian coordinates if proper attention is giver to the changing coordinate system of $k_{\perp}$ and $k_{11}$. Considerable simplification and insight may be obtained by taking advantage of the Fismiitonian nature of Eqs. (1) and (2) and using canonical variables ${ }^{7}$ Eirst applied to the tokamak geometry by wersinger, ott, and Finn. ${ }^{8}$ The spatial variables are $r, \theta, \phi$ where $r$ is the radius measured Erom the magnetic axis of the torus, $\theta$ is the poloidal angle and $\phi$ is the toroidal angle, which, in the case of an axisymmetric tokamak, does not appear in D. Conjugate wave number variables are $k_{r}, m=r k_{\theta}$ and $n=(R+r \cos \theta) k_{\phi}$, where $R$ is the major radius. in terms of these variables

$$
\begin{aligned}
& k_{11}=\left(k_{r} B_{r}+\left(\frac{m}{r}\right) B_{\theta}+\frac{n B_{\phi}}{(R+r \cos \theta)}\right) /|\underline{B}| \\
& k_{\perp}^{2}=k_{r}^{2}+\left(\frac{m}{r}\right)^{2}+\left(\frac{n}{R+r \cos \theta}\right)^{2}-k_{11}^{2},
\end{aligned}
$$

and the trajectory of the wave is defined by 


$$
\begin{array}{ll}
\frac{d r}{d t}=-\frac{\partial D / \partial k_{r}}{\partial D / \partial \omega} ; & \frac{d \theta}{d t}=-\frac{\partial D / \partial m}{\partial D / \partial \omega} ; \frac{d \phi}{d t}=-\frac{\partial D / \partial n}{\partial D / \partial \omega} \\
\frac{d k_{r}}{d t}=+\frac{\partial D / \partial r}{\partial D / \partial \omega} ; & \frac{d m}{d t}=+\frac{\partial D / \partial \theta}{\partial D / \partial \omega} ; \frac{d n}{d t}=+\frac{\partial D / \partial \phi}{\partial D / \partial \omega}=0 .
\end{array}
$$

Let us now specialize to a simple equilibrium model ir which $B_{r}=0, \quad B_{\theta}=(1 / 2 \pi r) \int_{0}^{r} \mu_{0} J\left(r^{\prime}\right) 2 \pi r^{\prime} d r^{\prime}, B_{\phi} \sim(R+r \cos \theta)^{-1}$ where $\mu_{0}$ is the permitivity of free space and $J$ is the current density. Magretic surfaces are circles concentric about the magnetic axis. With $B_{\theta} \ll B_{\phi}$, we have

$$
k_{11} \cong \frac{n}{R+r \cos \theta}\left(1+\frac{m}{n q}\right) \quad ; \quad q=\frac{r B_{\phi}}{(R+r \cos \theta) B_{\theta}} \text {. }
$$

Equation (12) shows that $k_{1}$ varies modestly with $(R+r \cos \theta)^{-1}$ if $m \ll n q$, but from Eq. (II) we can see that in varies along the trajectory. Using $k_{1}^{2} \gg k_{1}^{2}$ (appropriate to the problem) and ignoring electromagnetic and thermal effects, we find ${ }^{9}$

$$
\frac{d m}{\omega d t} \equiv-\frac{r \sin \theta}{R+r \cos \theta}\left(\frac{\omega_{p e}^{2} / \omega_{c e}^{2}}{1+\omega_{p e}^{2} / \omega_{c e}^{2}}+\frac{\left(1+\pi_{1} / n q\right)^{-1}}{1+\omega_{p e}^{2} / \omega_{c e}^{2}}\right) .
$$

From Eq. (13), and an examination of the sign of d $\mathrm{d} / \mathrm{dt}$, it becomes clear that a wave moving from small to large toroidal field suffers a decrease in $\left|k_{1}\right|$ [relative to the increase by $(R+r \cos \theta)^{-1}$ ] and vice versa. This is important since it is well known that accessibility of lower hybrid waves depends on $\left|k_{1}\right| .{ }^{3}$ Additionally, current generation by lower hybrid waves is strongly dependent on the $\mathrm{k}_{1}$ spectrum. 10 obviously, important details of the change in 
$k_{11}$ depend on details of profiles and the thermal and electromagnetic terms.

An estimate of the wave absorption can be found by adding imaginary parts to the dispersion relation according to the imaginary parts of the plasma dispersion functions ${ }^{11}$ contained in the expressions for $\underline{\underline{K}}(\underline{\underline{r}}, \underline{k}, w)^{3}$. The damping terms for the electrons and ions $\left(\mathrm{d}_{\mathrm{e}}, \mathrm{d}_{\mathrm{i}}\right)$ are

$$
\begin{aligned}
& a_{e}=2 \sqrt{\pi} \frac{\omega_{p e}^{2}}{\omega^{2}} k_{l l}^{2} I_{0}\left(\lambda_{e}\right) \exp \left(-\lambda_{e}\right) \xi_{o e}^{3} \exp \left(-\xi_{o e}^{2}\right), \\
& a_{i}=\sqrt{\pi} \frac{\omega_{p i}^{2}}{\omega^{2}} \sum_{\ell=-\infty}^{\infty} \frac{\omega^{2}}{v_{T i}^{2}} I_{\ell}\left(\lambda_{i}\right) \exp \left(-\lambda_{i}\right) \xi_{o i} \exp \left(-\xi_{\ell i}^{2}\right),
\end{aligned}
$$

where

$$
\lambda_{e, i}=k_{\perp}^{2} v_{T_{e, i}}^{2} / \omega_{c e, i}^{2} ; \quad \xi_{\ell_{e, i}}=\frac{w+\ell_{c e, i}}{\sqrt{2} k_{11} v_{T_{e, i}}}
$$

and I represents the modified Bessel function. The electromagnetic dispersion relation of Eq. (5) must be divided by $k_{\perp}^{2}$ to give proper scale to the damping terms. The ion term can be simplified with a Hadamard ${ }^{6,12}$ expansion for large $\lambda_{i}$ to the following ${ }^{6}$

$$
\begin{aligned}
& a_{\dot{i}}=2 \sqrt{\pi} \frac{\omega_{p i}^{2}}{\omega^{2}} k_{\perp}^{2} x_{i}^{3} \exp \left(-\chi_{i}^{2}\right)\left[\frac{\sum_{\ell\left(\omega_{c i} / \omega\right) \xi_{0 i} \exp \left(-\xi_{\ell i}^{2}\right)}}{\sqrt{\pi}}\right], \\
& x_{i}=\frac{\omega}{\sqrt{2} k_{\perp} v_{T_{i}}} .
\end{aligned}
$$

If $k_{11}$ is large enough that

$$
\frac{\omega_{c i}}{\sqrt{2} k_{i} v_{T_{i}}} \ll 1
$$


then many terms of the sum in square brackets in Eq. (16) are important, the sum can be approximated by an integral, and the term in square brackets is approximately equal to 1 . In this case the ion damping term looks like the electron damping, and the result is equivalent to treating the ions as unmagnetized. For our case the reverse inequality is true, and at any given location only one to a few of the harmonic terms are important.

However, since many different harmonics are crossed by the wave in traversing the plasma, a spatial average of the quantity in square brackets can be justified if the damping is not very strong, and the quantity in square brackets is again approximately equal to 1 . Then, a perturbation solution for the imaginary part of $k_{\perp}$ is $\left[\left(F(x)=x^{3} \exp \left(-x^{2}\right) ; \lambda_{e} \ll 1\right]\right.$.

$$
\frac{\operatorname{Im} k_{\perp}}{\operatorname{Re} k_{\perp}}=\frac{\sqrt{\pi}}{\partial D / \partial k_{\perp}}\left(F\left(\xi_{o_{i}}\right) \frac{\omega_{p e}^{2}}{\omega^{2}} \frac{k_{1}^{2}}{k_{\perp}^{2}}+F\left(x_{i}\right) \frac{\omega_{p i}^{2}}{\omega^{2}}\right)
$$

and an approximation for the decrease in wave power $P$ due to electron Landau damping and high harmonic cyclotron damping (which has the form of perpendicular ion Landau damping in the estimate used) is given by

$$
\mathrm{P} / \mathrm{P}_{\text {initial }}=\exp \left(-2 \int I m k_{\perp} \mathrm{dr}\right)
$$

In the numerical work to follow, the ray equations (11) are integrated with $\mathrm{D}$ given $\mathrm{E} \mathrm{Eq}$. (5), without treatment of damping. As the integration proceeds, damping estimates are accumulated according to Eqs. (17) and (18). 
In considering the calculated results, it is helpful to keep in mind the following picture of the way heating takes place in the linear theory without coroidal effects. ${ }^{4}$ A slow electrostatic wave is launched at the outside of the plasma and travels inward with

$$
k_{1}^{2} \cong \frac{k_{11}^{2}\left(-k_{11}\right)}{k_{1}}
$$

in the low temperature region. Near the mode conversion surface, on which $k_{\perp}^{2}=4 \alpha k_{\|}^{2}\left|k_{\mid}\right|$, the dispersion relation is doninated by thermal effects and

$$
\mathrm{k}_{1 .}^{2} \cong \frac{\mathrm{k}_{1}}{2 \alpha}
$$

The wave then propagates outward. Somewhere near the mode conversion, the wave can be damped by high harmonic cyclotron damping (or perpendicular ion Landau damping) if $k_{\perp} v_{\mathrm{Ti}} / \omega \sim 1$, according to the ion term in Eq. (17). Hot plasma effects, not found in the first finite Larmor radius terms of Eqs. (5) and (6), cause a second mode conversion with a change in wave direction again. If the density is low enough, there is no first mode conversion, $k_{\perp}$ never becomes very large, and there is no ion heating. Also, if $k_{11}^{2}$ is too low $\left[<\left(\omega^{2} / c^{2}\right) k_{1}\right]$, the slow electrostatic wave of Eq. (6) couples to the fast electromagnetic solution of Eq. (5) and $k_{\perp}$ never becomes large. Electrons can be heated by either the fast or the slow wave, with or without mode conversion of the latter, whenever $k_{11} v_{T e} / \omega \sim 1$. 


\section{Numerical Treatment}

Equations (11) are integrated by predictor-corrector routine with a Runge-Kutta starter. Derivatives by $\mathrm{k}_{\mathrm{r}}, \mathrm{m}, \mathrm{n}$ are found with explicit formulae, and derivatives by $r, \theta$ are found numerically. The deviation of $D$ from zero divided by the largest term in $D$ is found to remain small $\left(<10^{-4}\right)$. The ray is started on the slow wave solution at a radius slightly inside the layer $\omega_{p e}(r)=\omega$; outside of this layer the wave is evanescent. The $\theta$ location is usually set to 0 (outside) but has been set to other locations, especially $\pi / 2$ (top) and $\pi$ (inside). At that point $n$ is set to $(R+r \cos \theta) k_{1 \mid}(a)$, where $k_{1 \mid}(a)$ is imagined to be part of the spectrum of an external coupling device, $m$ is set to zero and $\mathrm{k}_{\mathrm{r}}$ satisfies the slow branch of the electromagnetic dispersion relation. The external coupler would, in general, excite m's from 0 to 10 or so, but since $m$ grows from 0 to $100^{\prime} s$ in the solution, intializing $m$ at zero seems a sufficient afproximatir . The electron (and ion) density profile is modeled by $n_{e}(0)=n_{e}(0) \cdot\left(1-r^{2} / a^{2}\right)$; the ion temperature profile by $T_{i}(r)=T_{i}(0) \cdot\left(1-r^{2} / a^{2}\right)^{2}$; the electron temperature profile by $T_{e}(0)=T_{e}(0) \cdot\left(1-r^{2} / a^{2}\right)^{1.4}$. The poloidal field is $B_{\theta}(r)=\left(\mu_{0} / 2 \pi r\right) I_{p l a s m a} \cdot\left[I-\left(I-r^{2} / a^{2}\right)^{q(a)}\right]$, which gives $q=1$ in the center and $\langle q(r)\rangle=q(a)$ at the outside. The expression

$$
q(a)=\frac{1}{200} \frac{a_{c m}^{2}}{R_{c m}} \frac{b_{T e s 1 a}}{I_{M A}}
$$

is used. 13 


\section{Pesults}

A good example of the toroidal effect on $k_{11}$, and thereby accessibility and power absoiption, can be seen in a case of nominal parameters for an experiment on PLT. We take $k_{1}(a) c / \omega=4.5$, $\mathrm{R}=135 \mathrm{~cm}, \mathrm{a}=40 \mathrm{~cm}, \mathrm{~B}_{,}(\mathrm{r}=0)=2.5 \mathrm{Tesla}, I_{\text {plasma }}=0.4 \mathrm{MA}$, $\omega / 2 \%=800 \mathrm{MHz}, \mathrm{n}_{\mathrm{e}}(0)=4 \times 10^{13} \mathrm{~cm}^{-3}, \mathrm{~T}_{\mathrm{e}}(0)=1.5 \mathrm{keV}, \mathrm{T}_{\mathrm{i}}(0)=0.8 \mathrm{keV}$, profiles as described previously, and assume a deuterium plasma.

Figure 1 shows (a) evolution of $k_{11}$, (b) path of the ray in the $r, \theta$ plane along with the position (dashed) of the linear mode conversion surface if $k_{\eta l}$ were forced to vary only as $(R+r \cos \theta)^{-1},(c)$ evolution of $k_{\perp},(d)$ an isometric view of the path of the wave in the torus. Parts (e) and (f), included for comparison to later figures, show that there is no damping decrement from either ions or electrons and that the wave amplitude is constant. (Recall that this estimate of the amplitude change ignores focusing and defocusing effects.) The important point of Fig. 1 is that the wave goes through the nominal mode conversior. surface, near which damping should occur, but because $k_{1}$ changes considerably, no effect on the amplitude is seen.

The calculation of Fig. I has been extended to long times and many toroidal circuits of the plasma; the wave leaves the torus on the fast mode without experiencing any damping. The effect revealed here, in which $k_{11}$ decreases as the wave moves to smaller major radius and mode conversion is avoided, depends on motion to small major radius $(\mathrm{R}+\mathrm{r} \cos \theta)$ rapid compared to motion small minor radius $r$. The effect is reduced by higher $R / a, q(a), k_{11}(a)$, $\mathrm{n}_{\mathrm{e}}(0)$, and $\mathrm{T}_{i}(0)$. For the particular parameters of $\mathrm{Fig} .1$, 
changing $\mathrm{n}_{\mathrm{e}}(0)$ from $4 \times 10^{13} \mathrm{~cm}^{-3}$, to $5 \times 10^{13} \mathrm{~cm}^{-3}$, or $\mathrm{q}(\mathrm{a})$ from 3.7 to 5 , or $R$ from $135 \mathrm{~cm}$ to $225 \mathrm{~cm}$ [at constant $q(a)$ ] results in a case in which the wave is absorbed. Figure 2 shows the result of changing $R$ to $225 \mathrm{~cm}$ [holding $q(a)$ constant]. In Fig. 2 the wave is absorbed by ion damping very near the position of the linear mode conversion surface found if $k_{11}$ is forced to vary only as $(R+I \cos \theta)^{-1}$. Accordingly, a toroidal calcula-tion sinilar to this one (electrostatic with thermal correction) by Ott, Wersinger, and Finn found mode conversion to persist for the $\mathrm{R} / \mathrm{a}=5, \mathrm{q}(\mathrm{a})=4$ case that was computed. 14

The two cases shown in Figs. 1 and 2 suggest that the range of parameters for which lower hybrid waves heat toroidal plasmas is smaller than the range predicted in a cylindrical (or planar) geometry.

If the lower hybrid slow wave is excited at the inside of the torus, $k_{1}$ will tend to rise, and it is probably true that the range of parameters for successful heating is little modified relative to a plane parallel model. Experimental difficulties with interior couplers make a study of these cases academic, however. More interesting is the result of launching the wave at the top (or bottom) of the torus. In such a case, a symmetric excitation will launch two waves of qualitatively different behavior, as seen in Figs. 3 and 4 . In these cases, a low density is chosen to isolate damping by electrons, and investigate the possibility for current drive by a symmetric coupler. The outward-going wave of Fig. 3 couples with electrons, whereas the inward-going wave of Fig. 4 does not. 
IV. Conclusion

Toroidal effects on lower hybrid wave propagation are important, especially for low aspect ratio and low q. The exact behavior depends critically upon profiles, but qualitatively the toroidal effect is to modify the $k_{1}$, and therefore electron damping and change the evolution of $k_{1}$, and therefore ion damping. For practical configurations, the range of parameters allowing heating is smaller in a torus thar. in a cylinder, perhaps significantly so.

Toroidal effects are symmetric about the mid-plane, and therefore asymmetric for waves excited at the top (or bottom) of the torus, suggesting the possibility of current arive with a symmetric coupler. However, current drive situations depend on control of the $k_{1}$ spectrum inside the plasma, which is complicated by the role a toroidal plasma plays in modifying the $k_{11}$.

\section{ACKNOWLEDGMENT}

This investigation was suggested by Dr. W.M. Hooke, and was aided by numerous discussions with him and others at Princeton interested in rf heating, especially Drs. J.C. Hosea, P.I. Colestock, D. Hwang, S. Bernabei, and R.W. Motley. Dr. J. Stevens collaborated with the author on portions of the computer program.

This work was supported by the United states Department of Energy Contract No. DE-AC02-76-CHO3073. 
Note Added in Proof

Results qualitatively similar to those reported in this paper have been reported recently by T. Maekawa, Y. Terumichi, and 5. Tanaka. ${ }^{15}$ Their paper includes results from the cold electromagnetic dispersion relation [Eq. (5) with $\alpha=0$ ] and a hot plasma electrostatic dispersion relation different in detail from Eq. (6). 
REFERENCES

1. S. Weinberg, Phys. Rev. Lett. 126, 1899 (1962).

2. I. B. Bernstein, Phys. Fluids 18, 320 (1975).

3. T. H. Stix, The Theory of Plasma Wave (McGraw-Hill, New York, 1962).

4. T.H. Stix, Phys. Rev. Lett. 15, 878 (1965).

5. V. M. Galgolev, Plasma Phys. 14, 301, 315 (1972).

6. M. Brambilla, Plasma Phys. 18, 669 (1976).

7. This approach was suggested by E. Valeo (private communications).

8. J. M. Wersinger, E. Ott, J. M. Finn, Phys. Fluids 21, 2263 (1978).

9. A similar formula appears in P. L. Colestock and J. L. Kulp, IEEE Trans. on Plasma Sci., Vol. PS-8, 71 (1980).

10. N. J. Fisch, Phys. Rev. Lett. $\underline{41}, 873$ (1978).

11. B. D. Fried and S. D. Conte, The Plasma Dispersion Function (Academic, New York, 1961).

12. J. P. Schmidt, J. Plasma Phys. 12, 51 (1974).

13. Profile forms were suggested by F. J. Paoloni as an appropriate model for PLT discharges (private communications).

14. E. Ott, J.-M. Wersinger, P. T. Bonoli, Phys. Fluids 22, 192 (1979).

15. T. Maekawa, Y. Terumichi, and S. Tanaka, IEEE Trans. on Plasma Science, vol. PS-8, 67 (1980). 

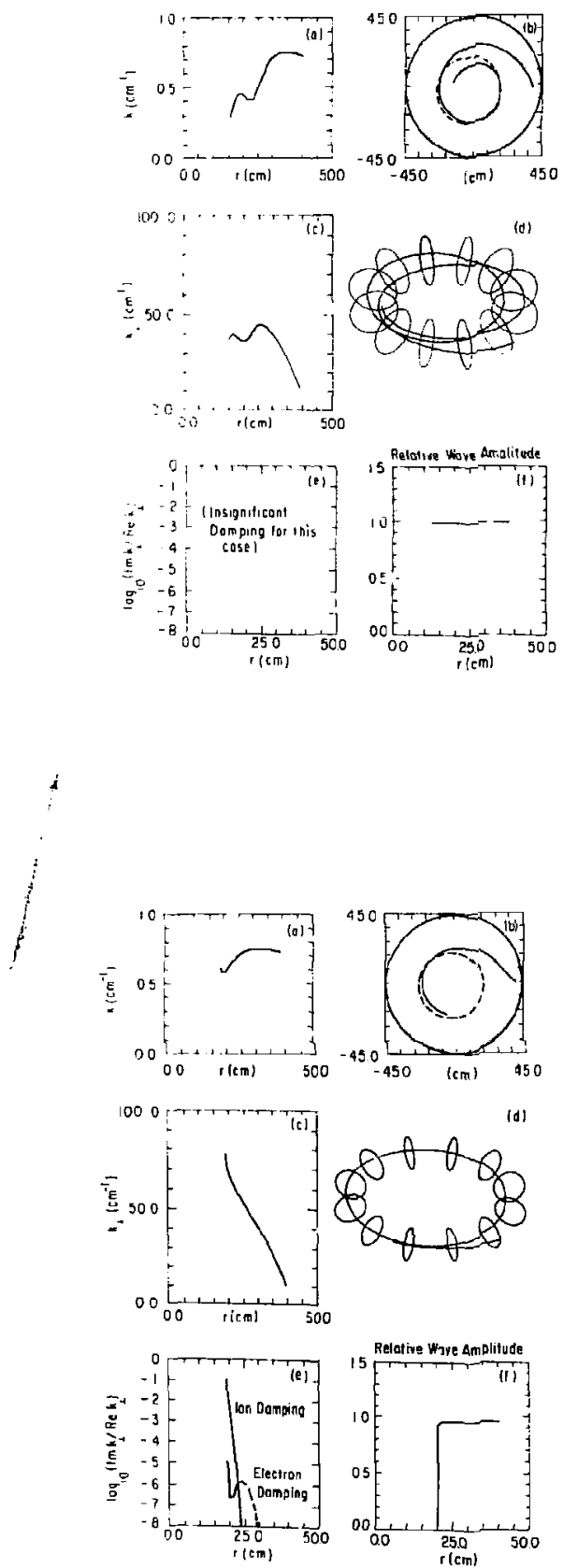

(PPPL-806372)

Fig. 1. Evolution of ray parameters for the PLT model described in the text. Density is $4 \times 10^{13} \mathrm{~cm}^{-3}$. Part (a), $k \|\left(\mathrm{cm}^{-1}\right)$ versus minor radius. Part (b), ray trajectory in the $r, \theta$ plane (solid) and location of the linear mode conversion surface (dashed)

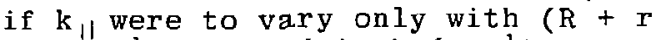
$\cos \theta)^{-1}$. Part (c), $k_{1}\left(\mathrm{~cm}^{-1}\right)$ versus radius. part (d), isometric view of ray path in the torus. Part (e), log ( Im $k_{1} / \operatorname{Re} k_{1}$ ) when the $\log$ is greater ${ }^{10}$ that -8; in this case log of the damping ratio is less than -8 . Part (f), radial evolution of estimated relative power in the wave.

(PPPL-806373)

Fig. 2. Repetition of the case in Fig. 1, except that $R=225 \mathrm{~cm}$ and 1 . In this case mode chnvermation occurs and the wave is absorbed primarily by ions. All parts are the same as in Fig. 1. 

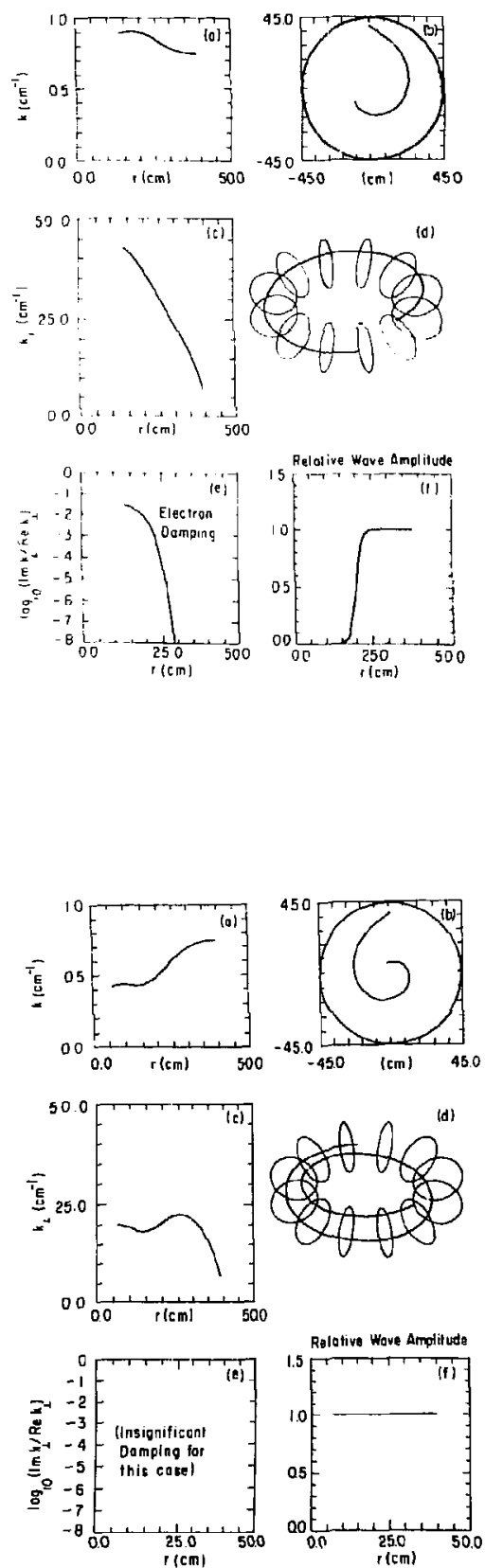

(PPPL-806371)

Fig. 3. Examination of a low density case $\left[\mathrm{n}(0)=1.5 \times 10^{13} \mathrm{~cm}^{-3}\right]$ with the wave started at the top of the plasma toward larger $(R+r \cos \theta)$. Parts (a) - (f) show the same kind uf information described in Figs. 1 and 2. Coupling to the electrons is strong.
(PPPL-806370)

Fig. 4. As in Fig. 3, but the wave is started toward smaller $(R+r \cos \theta)$. There is no coupling to electrons. 